\title{
Explorative visual analytics on interval-based genomic data and their metadata
}

\author{
Vahid Jalili ${ }^{*}$ (D) Matteo Matteucci, Marco Masseroli and Stefano Ceri
}

\begin{abstract}
Background: With the wide-spreading of public repositories of NGS processed data, the availability of user-friendly and effective tools for data exploration, analysis and visualization is becoming very relevant. These tools enable interactive analytics, an exploratory approach for the seamless "sense-making" of data through on-the-fly integration of analysis and visualization phases, suggested not only for evaluating processing results, but also for designing and adapting NGS data analysis pipelines.

Results: This paper presents abstractions for supporting the early analysis of NGS processed data and their implementation in an associated tool, named GenoMetric Space Explorer (GeMSE). This tool serves the needs of the GenoMetric Query Language, an innovative cloud-based system for computing complex queries over heterogeneous processed data. It can also be used starting from any text files in standard BED, BroadPeak, NarrowPeak, GTF, or general tab-delimited format, containing numerical features of genomic regions; metadata can be provided as text files in tab-delimited attribute-value format. GeMSE allows interactive analytics, consisting of on-the-fly cycling among steps of data exploration, analysis and visualization that help biologists and bioinformaticians in making sense of heterogeneous genomic datasets. By means of an explorative interaction support, users can trace past activities and quickly recover their results, seamlessly going backward and forward in the analysis steps and comparative visualizations of heatmaps.
\end{abstract}

Conclusions: GeMSE effective application and practical usefulness is demonstrated through significant use cases of biological interest. GeMSE is available at http://www.bioinformatics.deib.polimi.it/GeMSE/, and its source code is available at https://github.com/Genometric/GeMSE under GPLv3 open-source license.

Keywords: Genomic data analysis, exploration, visualization, Interactive and visual analytics, Comparative evaluation, Next Generation Sequencing

\section{Background}

High-throughput sequencing technologies generate high amounts of genomic, epigenomic and transcriptomic data regarding multiple genomes in different conditions. Complex pipelines are used for selecting high-quality sequenced raw data, aligning them to a reference genome, and then calling specific features on the aligned data, such as DNA mutations, transcription factor bindings, histone modifications, DNA methylations, gene expressions $[1,2]$. Thanks to large international consortia (e.g., Encyclopedia

*Correspondence: vahid.jalili@polimi.it

Dipartimento di Elettronica, Informazione e Bioingegneria, Politecnico di Milano, 20133 Milano, Italy of DNA Elements (ENCODE) [3], Roadmap Epigenomics [4], The Cancer Genome Atlas (TCGA) [5], and the 1000 Genomes Project [6]), such data are organized within open repositories, which provide easy access to raw and processed datasets. The availability of these datasets is reshaping modern biology: researchers can complement their own experimental datasets with a large body of public data and knowledge, and can derive relevant results which are just based upon secondary analysis of open data.

GenoMetric Query Language (GMQL) [7] is an innovative cloud-based system to efficiently compute arbitrarily complex queries over heterogeneous processed datasets, taking into account both genomic region features 
and sample global characteristics (i.e., metadata). GMQL queries apply to genomic datasets of Next Generation Sequencing (NGS) processed data to extract interesting data samples and their genomic regions and metadata; such valuable GMQL output needs further data exploration and analysis to support biological interpretation of results.

This paper presents a rich set of abstractions for data analysis, exploration and visualization, and their implementation in an associated tool, named GenoMetric Space Explorer (GeMSE); GeMSE supports primitives for data explorations spanning from select, sort, and discretize, to clustering, and pattern extraction. GeMSE seamlessly manages metadata together with genomic region data and shows them aggregated for any of the result clustering patterns. GeMSE leverages on GMQL as its back-end tertiary data retrieval framework, but can be used on any text files in standard BED (Browser Extensible Data), BroadPeak, NarrowPeak, GTF (General Transfer Format), or general tab-delimited format, containing data regarding features of genomic regions; metadata can also be provided as text files, in tab-delimited attribute-value format.

Genomic data visualization builds on two orthogonal concepts: genome browsing and quantitative visualization. A genome browser, pioneered by Artemis [8] and popularized by the University of California at Santa Cruz (UCSC) Genome Browser [9], is commonly used for looking at genome features within a given portion of the genome. In the realm of quantitative visualizations, clustering techniques and heatmaps (proposed outside biology) were used by Eisen and colleagues [10] for the evaluation of microarray gene expression data; they have been implemented in some stand-alone tools (e.g., GENE-E [11]) and they are supported in many statistical software, including Matlab, Mathematica and $\mathrm{R} /$ Bioconductor [12], as well as scripting languages such as Python. Lately, they have been applied to NGS data, and implemented within a few tools specifically devoted to such data (e.g., seqMINER [13], ngs.plot [14], or MicroScope [15]). These tools are mainly designed to be used on NGS raw or aligned data; unless they are executed on very powerful servers, they can handle only a few data files at a time, limiting the possibility of quickly comparing multiple conditions and datasets simultaneously.

GeMSE can be regarded as enabler of interactive analytics (IA), a promising exploratory approach for the seamless "sense-making" of data through on-the-fly integration of analysis and visualization tools. Interactive analysis is suggested not only for evaluating processing results, but also for designing and adapting NGS data analysis pipelines. Remarkably different results could be produced with slightly different parameter settings of data production pipelines (e.g., for feature calling); choosing a "correct" parameter setting commonly breaks down to a difficult cycle of repeatedly tweaking parameters, re-running the analysis, and visually inspecting the results. Tweaking the parameters of the tools used for data generation is context-specific and could consist of tweaking parameters of GMQL scripts or Galaxy workflows [16]; other examples of IA frameworks include Cytosplore [17], focused on mass cytometry data for immune systems cellular composition studies, or Trackster [18], which leverages Galaxy's comprehensive data analysis framework (spanning from primary to tertiary analysis).

Data exploration is well supported by application suites such as Mathlab, Mathematica, Maple or SageMath (in Python), or scripting languages such as Python, R, Perl, or even shell scripting; however, not everyone has the required scripting/coding ability. GeMSE enables data exploration using intuitive visual interfaces for everyone, without need for any scripting, making data exploration seamless.

A key component of explorative data analysis, is to be able to perform actions in a non-sequential and repeatable way. To enable such data exploration, GeMSE adapts a state-space graph model, where nodes/states are the data and transition are the actions performed on the data. Users can choose any node, and perform any number of actions on a node (hence creating a new node), while all nodes are efficiently cached in memory, enabling the creation of (theoretically) an unlimited number of states. In general, every action by the user generates a new state/node, which can then be used in subsequent analyses, downloaded, or visualized. Nodes are immutable, i.e., once a node is generated, it cannot be changed (changes happen as new nodes). A key advantage of this feature is that if the user makes a mistake or wants to experiment with different parameter settings, he/she can always go back to the original data.

\section{Implementation}

Datasets in GMQL consist of one or more items, called samples, each of them associated with one experimental condition; each sample, in turn, consists of data and metadata. Data are genomic regions, expressing the result of a calling process that extracts genomic features (e.g., DNA mutations, gene expression scores, peaks of binding enrichment, epigenetic modifications) from measured (epi)genome signals. Metadata are attribute-value pairs expressing arbitrary properties of samples (e.g., the related tissue or cell-line, the technology used to obtain it, the experimental method applied; if the sample is human, it may include phenotypical information, such as the donor's sex, age and disease status).

\section{Genometric space}

A genometric space is produced by a specific GMQL operation, called MAP [7], which applies to two datasets, 
denoted as reference and experiment (see panel b on Fig. 1):

- The reference dataset consists of a single sample; it typically includes genomic regions corresponding to genes or exons, representing the coding portions of the genome, or transcription regulatory regions; however, the reference sample can be an arbitrary set of regions from the genome, possibly extracted by means of GMQL queries.

- The experiment dataset consists of multiple, possibly heterogeneous, samples, each constituted by multiple regions (similar to heterogeneous tracks that can be observed on a genome browser); experiment samples can be produced by different sources, while we expect each experiment sample to be produced by a single source.

The MAP operation produces a matrix structure, called genometric space, where each row is associated with a reference region, each column refers to a sample, and each matrix entry is computed by means of an aggregate function applied to the values of a selected attribute of the experiment regions of the sample that overlap the reference region (see panel c on Fig. 1). Formally:

- The MAP operation applies to a reference sample $R$ and to several experiment samples $S_{j}$, and has two parameters: an attribute $A$ of the regions of $S_{j}$ and an aggregate function $G$.

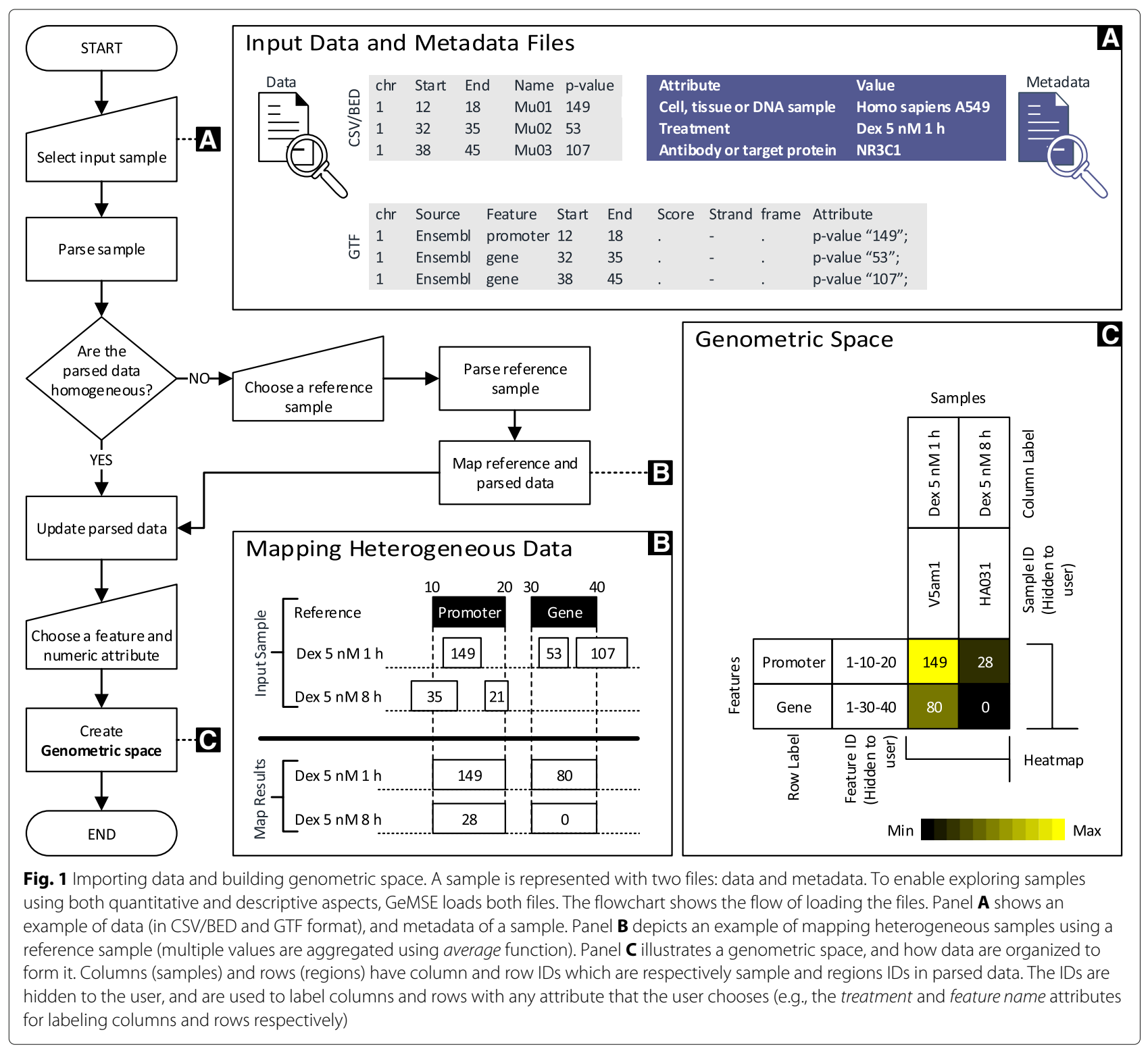


- The result of the MAP operation is a matrix $M$, whose entries $m_{i, j}$ are each built from the region $r_{i}$ of the reference and the sample $S_{j}$ of the experiment dataset by considering all regions $r_{k, j}$ of the $S_{j}$ sample having a nonempty intersection with $r_{i}$, then considering the bag (i.e., set) $B_{i, j}$ of all the values $v_{k, j}$ that the attribute $A$ gets for the $r_{k, j}$ regions, and then applying the aggregate function $G$ to $B_{i, j}$.

We support the classic aggregate functions COUNT, MIN, MAX, SUM, AVERAGE, and MEDIAN; COUNT is used to count the number of experiment sample regions intersecting a reference region, and requires no indication of a specific attribute. The $2 \times 2$ matrix in panel c on Fig. 1 represents 2 genomic regions and 2 experiment samples; values are $((149,28),(80,0))$. The matrix is organized in GeMSE with the reference regions as rows and the experiment samples as columns; this choice is preferred because there are typically many more regions than experiments.

When GeMSE is used in pipeline with GMQL, it reads the output of a GMQL MAP operation directly; when instead GeMSE is used as a stand-alone tool, it starts by applying a MAP operation to the reference and experiment samples specified by the user (see flowchart, panel $a$, and panel b on Fig. 1). Input region data can be read as formatted according to the standard BED, BroadPeak, NarrowPeak, or GTF formats, or in the form of a general BED-like tab-delimited format. Required fields of each region are chromosome (i.e., chr), start, and end, as in the BED format. Additional fields are considered as referenced by the correspondent input column header; e.g., GTF files in addition contain the fields source, feature (i.e., feature name), score, strand, frame, and a group field which is a text string containing a set of attribute-value pairs separated by a single space. Metadata can also be provided as separate tab-delimited text files, having the same name as the sample file to which they refer to, and an extension ".meta", storing items in a pair of fields, respectively called attribute and value. The flowchart in Fig. 1 shows that files of heterogeneous formats can be given in input to GeMSE.

\section{Interactive data exploration model}

GeMSE data exploration consists of three iterative phases, illustrated on Fig. 2 and explained as it follows:

- Transition, where a transformation function is applied on a genometric space resulting in a new genometric space.

- Analysis, where a genometric space is analyzed using data analysis functions (e.g., pattern analysis, or statistical inference).

- Visualization, where a genometric space is visualized (e.g., on heatmaps or graph views).

In GeMSE, genometric spaces are immutable and independent from each other; in other words, once a genometric space is created, it cannot be changed. Therefore, to enable data exploration, GeMSE organizes genometric spaces on a state-transition tree, explained in the following section. The genometric space transitions and analysis are explained the subsequent sections.

\section{State-transition tree}

Tracking multiple transformations of genometric spaces is crucial for data exploration. GeMSE tracks such transitions in a graph data structure called State-Transition Tree (STT), whose nodes represent different genometric spaces and whose edges represent the transformations between genometric spaces (e.g., see Fig. 3). From any data exploration state, one can view the related genometric space, visualizing it as a table or a heatmap, and also explore contained patterns (e.g., see Fig. 7, where the heatmaps labeled A1-A 5 and the associated pattern exploration refer to the first sequence of nodes on Fig. 3). STT visualization facilitates data exploration state examination and a trial-and-error approach.

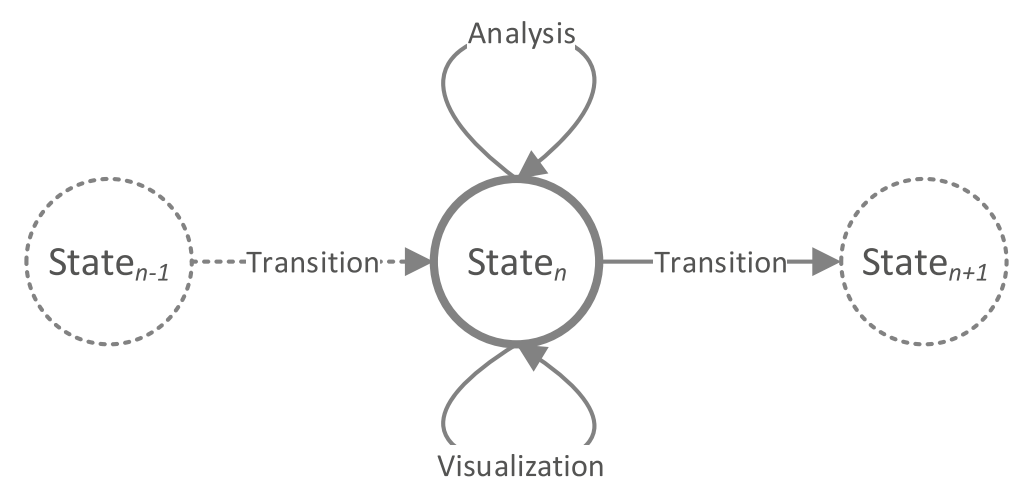

Fig. 2 The data exploration model of GeMSE 


$$
\begin{aligned}
& \text { Root } \\
& \text { Sort by treatment } \\
& \text { Extract EtOH } 0.02 \% \text { treatment samples } \\
& \text { Sort genes by p-value } \\
& \text { Discretize as weak, middle, strong significance } \\
& \text { Extract Dex treatment samples } \\
& \text { Sort genes by p-value } \\
& \text { Extract protein binding enriched genes }
\end{aligned}
$$

Fig. 3 An example of GeMSE State-Transition Tree; it represents the use-cases illustrated in the demonstration and discussion section of the paper

GeMSE stores nodes and edges of STT in memory. However, keeping all the nodes in memory is not an efficient practice, specially if the STT and genometric spaces are considerably large. Therefore, GeMSE implements the least recently used caching algorithm [19]. Accordingly, GeMSE stores the first data exploration state (i.e., the root of the STT), the genometric space of the $n$ most recent states (with the $n$ value being user modifiable), and the transitions of all the states. Least recently used states are removed from the memory, and if needed they are reconstructed. This is done first by recursively traversing the STT from the node to be reconstructed to the closest cached parent node; then, once the closest cached parent node is determined, the requested node is reconstructed by applying the stored transitions from the closest cached parent node to the requested one. Given that clustering is computationally expensive, dendrograms, i.e., cluster hierarchical structures, are always kept in memory to prevent cluster reconstruction.

\section{State transitions}

A state transition takes a state and some arguments as input, and generates a new state as output. In our case, a state transition is a data transformation performed during data exploration, and a state represents the explored data, in case resulting from one of such transitions. The general data transformations most useful in data exploration, which we implemented in GeMSE, are: Extract, Rewrite, Discretize, Sort, Cluster, and $\mathrm{Bi}-\mathrm{Cl}$ uster. In what follows, we give a semi-formal description of each of such state transitions as a genometric space transformation. It is important to note that these operations are specified in a very simple way by using the GeMSE tool, with an easy-to-use graphical interface that prompts, for each transformation, the parameters to be interactively entered.

\section{Extract}

This transformation extracts a sub-space $S^{\prime}$ of a genometric space $S$, given two ranges of columns and rows. Let $\left[C_{l}, C_{r}\right.$ ) and $\left[R_{u}, R_{d}\right.$ ) denote ranges for columns (with left and right bounds) and rows (with up and down bounds), respectively (inclusive lower-bound, exclusive higher-bound); the transformation is defined as follows:

$$
\begin{aligned}
S^{\prime}= & \text { Extract }( \\
& {\left[C_{l}, C_{r}\right), } \\
& {\left.\left[R_{u}, R_{d}\right)\right) } \\
& S
\end{aligned}
$$

After an Extract operation, the new state in the STT holds a new genometric space $S^{\prime}$, which is a subset of the input state $S$ (represented in light blue in panel a on Fig. 4). The data and metadata of the selected samples/rows are not changed, while the data and metadata of excluded samples/regions are discarded at the new state.

\section{Rewrite}

This transformation maps the values of an input genometric space $S$ into new values in a new genometric space $S^{\prime}$; if only a portion of $S$ where to apply the transformation is selected, all the other values of $S$ outside the selected portion remain unchanged, and the dimensions of $S^{\prime}$ are not modified with respect to those of $S$. The values of

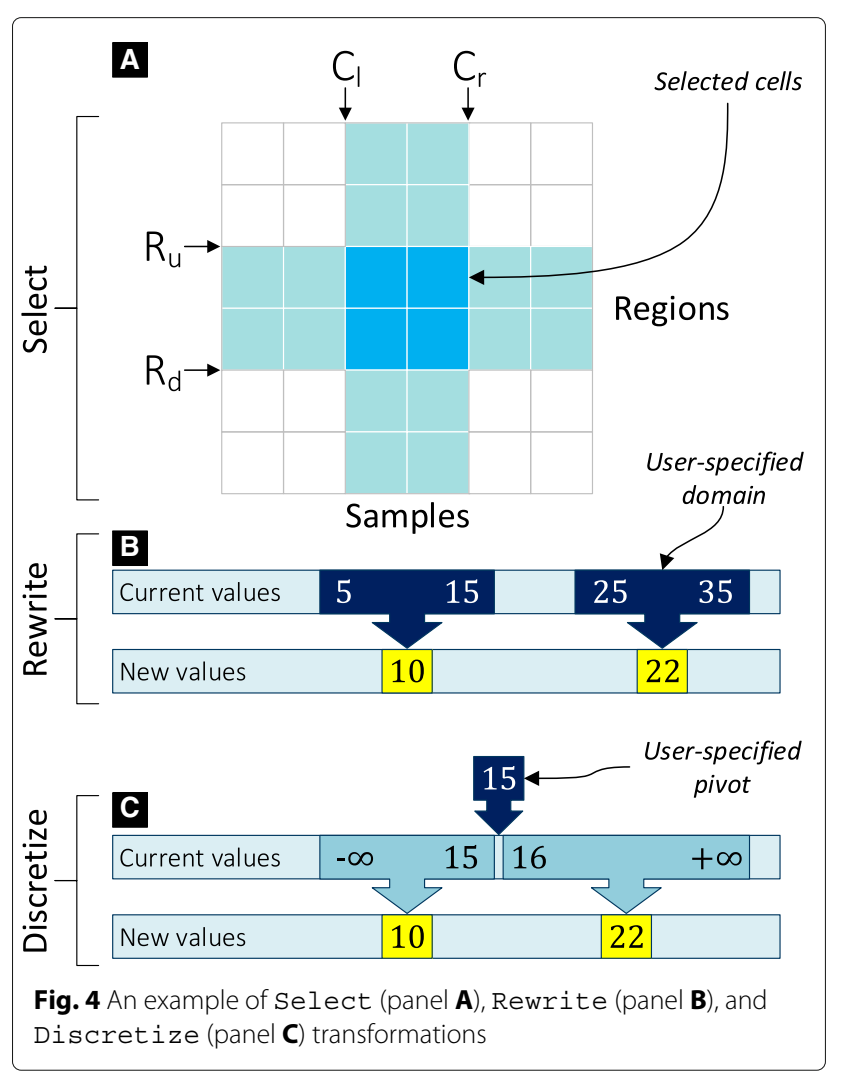


$S$ are mapped conditionally; the values of cells $\left[C_{l}, C_{r}\right)$, $\left[R_{u}, R_{d}\right)$ are mapped to a constant $V$, or $\log _{n}$ transformed (user-defined $n$ ), if the values are within the $\left[V_{\min }, V_{\max }\right]_{i}$ range. Several ranges may be used in the same Rewrite transformation, provided that the ranges do not overlap (e.g., see panel b on Fig. 4). Rewrite is a discrete mapping, such that the ranges not necessarily cover all the values in the input genometric space; the excluded values remain intact. Each value is changed based on the range that it falls in, e.g., $\left\{\left[V_{\min }, V_{\max }\right]_{1} \rightarrow V_{1},\left[V_{\min }, V_{\max }\right]_{2} \rightarrow\right.$ $\left.V_{2}, \ldots\right\}$. The transformation is defined as follows:

$$
\begin{aligned}
S^{\prime}= & \text { Rewrite }( \\
& {\left[C_{l}, C_{r}\right), } \\
& {\left[R_{u}, R_{d}\right), } \\
& \left.\left(\left[V_{\min }, V_{\max }\right],\left[V \mid \log _{n}\right]\right)^{+}\right) \\
& S
\end{aligned}
$$

\section{Discretize}

This transformation maps all the values of an input genometric space $S$ to new values in a new genometric space $S^{\prime}$, in case selecting only a portion of $S$ where to apply the transformation. The difference between the Rewrite and Discretize transformations is that Rewrite is a discrete mapping of values, whereas Discretize is a contiguous mapping; accordingly, the transformation ranges are specified differently (see panels $\mathrm{b}$ and c on Fig. 4). In Rewrite, users explicitly define the ranges $\left[V_{\min }, V_{\max }\right]_{i}$, which are user-defined independent ranges and not necessarily contiguous. Conversely, in Discret ize, users define transformation ranges implicitly, by using break values (pivots) $\left[V_{\text {pivot }}\right]_{i}$, based on which the transformation ranges are determined automatically. For instance, referring to panel c on Fig. 4, suppose the Discretize transformation operates on Natural numbers, and takes the pivot value 15 and the new values 10 and 22; then, the Discretize transformation automatically defines the ranges $(-\infty, 15]$ and $[16,+\infty)$, and maps the values in these two ranges to 10 and 22, respectively. Note that when this transformation operates on real numbers, the ranges around a pivot value $V_{\text {pivot }}$ are as $\left(-\infty, V_{\text {pivot }}\right]$ and $\left(V_{\text {pivot }},+\infty\right)$.

The Discretize transformation has also a NoChange option, which indicates that the values within a given range should not be changed. The transformation is defined as follows:

$$
\begin{aligned}
S^{\prime}= & \text { Discretize }( \\
& {\left[C_{l}, C_{r}\right), } \\
& {\left[R_{u}, R_{d}\right), } \\
& \left.\left(V_{\text {pivot }},\left[V_{\mathrm{b}} \mid \text { NoChange }\right],\left[V_{\mathrm{a}} \mid \text { NoChange }\right]\right)^{+}\right) \\
& S
\end{aligned}
$$

where $V_{\mathrm{b}}$ and $V_{\mathrm{a}}$ are the values with which the values before and after the $V_{\text {pivot }}$ value are respectively replaced.

\section{Sort}

This transformation sorts the rows or columns $(\mathrm{R} \mid \mathrm{C})$ of an input genometric space $S$ in ascending/descending order, based on the values of a list of region attributes (e.g., count, $p$-value), or of sample metadata (e.g., antibody target, disease), and stores the ordered result in a new genometric space $S^{\prime}$. The transformation is defined as follows:

$$
\begin{aligned}
S^{\prime}= & \operatorname{Sort}( \\
& {[\mathrm{R} \mid \mathrm{C}], } \\
& {[\text { ASCENDING } \mid \text { DESCENDING }], } \\
& {\left[\left(\text { Region Attribute }^{+} \mid\left(\text {Sample Metadata }^{+}\right]\right)\right.} \\
& S
\end{aligned}
$$

\section{Cluster}

This transformation executes the clustering of either rows or columns $(\mathrm{R} \mid \mathrm{C})$ of an input genometric space $S$, and produces as output a clustered genometric space $S^{\prime}$, as well as a dendrogram (hierarchical description of the various clustering steps) and a heatmap, that plots the genometric space sorted based on the dendrogram. The Cluster transformation performs agglomerative hierarchical clustering by single, average, or complete linkage (SINGLE | AVERAGE | COMPLETE), using distance and correlation metrics; GeMSE implements Euclidean (EU), Manhattan (MA), Earth Movers (EA), Chebyshev $(\mathrm{CH})$, and Canberra (CA) distance metrics, and Pearson correlation ( $\mathrm{PE}$ ) metrics. The transformation occurs by first producing the clustering dendrogram, and then using the dendrogram for sorting the genometric space rows (regions) or columns (samples). The transformation is defined as follows:

$$
\begin{aligned}
S^{\prime}= & \text { Cluster }( \\
& {[R \mid C], } \\
& {[\text { S INGLE } \mid \text { AVERAGE } \mid \text { COMPLETE }], } \\
& {[\text { EU } \mid \text { MA } \mid \text { EA } \mid \text { CH } \mid \text { CA } \mid \text { PE }]) } \\
& S
\end{aligned}
$$

\section{Bi-cluster}

This transformation clusters both rows and columns simultaneously of an input genometric space $S$. To implement it in GeMSE, we used the R package hclust [20] (see "Availability and requirements" section), which performs bi-clustering by complete linkage (COMPLETE) using the Euclidean (EU) distance metrics. GeMSE automatically creates a script to be executed in R, then runs the script, and finally imports the generated result (i.e., a heatmap in .png format). Thus, the Bi-Cluster transformation in GeMSE does not generate a state that can be used 
for further transitions, since GeMSE has access to the clustering output of $\mathrm{R}$ as a heatmap only. The generated heatmap (i.e., output genometric space representation) is therefore a leaf node of the state-transition tree. The transformation is defined as follows:

$$
\begin{aligned}
S^{\prime}= & \text { Bi-Cluster( } \\
& {[\text { COMPLETE }], } \\
& {[\mathrm{EU}]) } \\
& S
\end{aligned}
$$

GeMSE supports other transformations performed by means of R packages; some of them (e.g., gplots [21]) require first a normalization of the distances of the clustering dendrogram from the leaves to the root; then, the updated dendrogram is exported to $\mathrm{R}$ in Newick tree format [22], along with the genometric space on which to apply it and the $\mathrm{R}$ script to be run. All these transformations with R-based implementations produce only the heatmap representation of the output genometric space; thus, in the state transition tree all of them generate a leaf node only, which is not usable for further transitions.

\section{State analysis}

An analysis function takes a state, and executes data analysis function on it. GeMSE implements two commonly used class of data analysis functions: pattern extraction, and statistical inference (e.g., statistical hypothesis testing, or principal component analysis), briefly described in the following sections.

\section{Pattern extraction}

A relevant task in data exploration concerns with the identification of patterns in the data, and their association with specific data aspects (e.g., biological features, supporting biological interpretation of the results).

Within a data matrix (i.e., genometric space), a pattern can be defined as an ensemble of feature values associated with a group of rows/columns which are similar based on such values. These patterns can be discovered through the Cluster data transformation implemented in GeMSE, by using either distance (e.g., Euclidean or Manhattan distance) or correlation (e.g., Pearson correlation) metrics between vectors of rows/columns containing such feature values; these vectors are clustered hierarchically, and patterns are extracted by cutting the clustering dendrogram at a given height. By doing so, the nearest (most similar) vectors of rows/columns are grouped together, unveiling a pattern. Patterns can then be explored in GeMSE by means of:

- Heatmaps, which effectively visualize each pattern (e.g., panel a on Fig. 5 and panel A5pc on Fig. 7).

- Radial graph [23], where nodes are the pattern analysis vectors (columns or rows of the genometric space), and edges are the relations between vectors. The visualization is interactive, it enforces a radial ordering of the nodes, while keeps a user-selected node at the center. Additionally, if selected by the user, it can color nodes differently, based on the pattern analysis result (see panel b on Fig. 5).

- Force-directed graph [23]; it is an interactive visualization forcing a graph view, which can aggregate nodes belonging to the same pattern (user-selected, see panel c on Fig. 5).

- Vectors forming the pattern, displayed in forms of heatmaps (e.g., panels A2p0, A2p1, and A2p2 on Fig. 7), or tabular views of vector values or metadata (e.g., the table on Fig. 7).

- Metadata counts, representing the aggregated occurrences of each metadata attribute-value pair in each pattern (e.g., the table on Fig. 9); they facilitate the identification of common/exclusive metadata within each pattern, and the interpretation of patterns based on such metadata.

\section{Number of clusters}

A key aspect in the described pattern extraction strategy is the choice of where cutting the dendrogram so as to identify an ideal number of patterns. GeMSE can suggest the best number of clusters; it does so by taking advantage of the clustering dendrogram produced by the Cluster data transformation, and by using the Elbow method [24]. This method compares the sum of squared distances between clusters for different number of clusters, plotted against the number of clusters; the optimal number of clusters is determined by identifying an "elbow" in the plot. To identify it, we first determine the total variance of the distances between the children of all nodes in the clustering dendrogram (i.e., between all clusters). Then, we calculate the variance percentage as the variance of the distances between the children of the nodes in the dendrogram (i.e., between clusters) at different dendrogram cutting heights (i.e., for different number of clusters), divided by the total variance. Finally, we compare the slope of two consecutive points in the plot (i.e., the variation of variance percentage for two consecutive dendrogram cutting heights, that is for two consecutive numbers of clusters): an "elbow" is where the difference of slopes between consecutive points is maximum (see Fig. 8). The pseudocode of the method is given in Algorithm 1.

Several other methods exist to determine the best number of clusters, based on gap statistic [25], or on "stopping rules" [26], or exploiting the Direction Division Partitioning principle [27] (i.e., stopping partitioning when centroid scatter value exceeds the maximum cluster scatter value at any node in the clustering dendrogram). Other methods are based on maximizing the distance between 


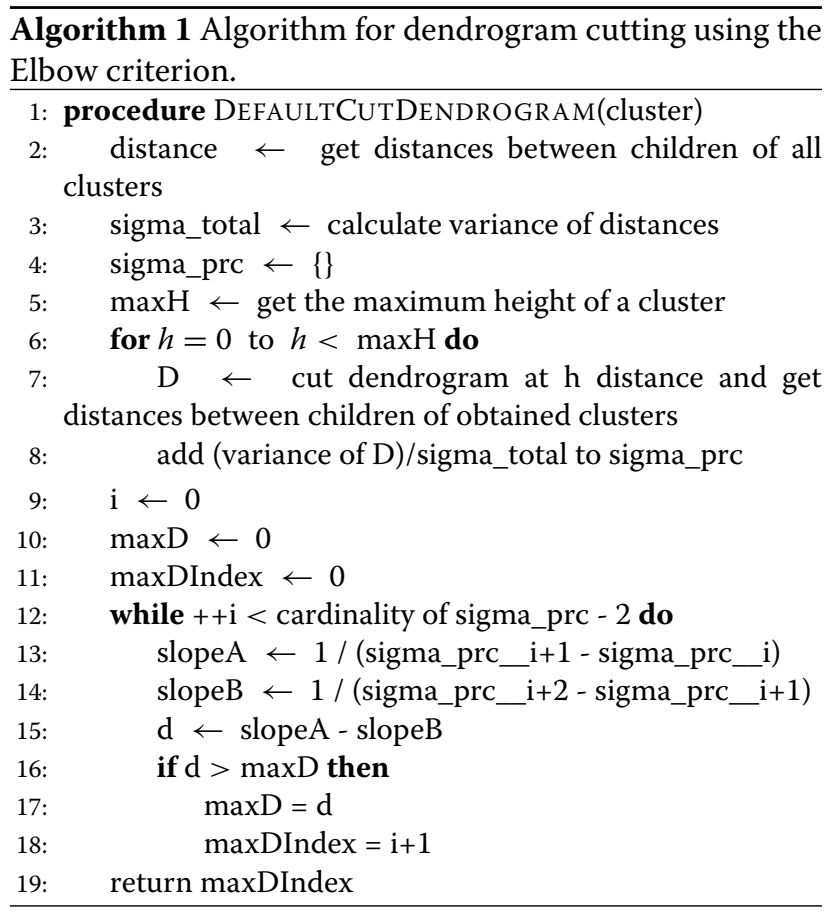

patterns and relative closeness [28], or on information criterion approaches - such as Akaike information criterion [29], Bayesian information criterion [30], or Deviance information criterion [30]. Note that no method performs always well; particularly, the Elbow method does not work well if the data are not very clustered. The GeMSE user can always interactively define the number of clusters to consider.

\section{Statistical inference}

Samples (columns) or regions (rows) of a genometric space can represent results of different hypothesis testing (e.g., DNA-protein binding significance); hence, GeMSE implements commonly used statistical inference methods to test (null and alternative) hypothesis, deduce properties, and evaluate correlation and dependencies between samples or regions. The methods for statistical inference implemented in GeMSE follow in the following two classes:

- Statistical hypothesis testing: GeMSE allows the hypothesis testing based on the following statistics computed for a genometric space: t-statistic, one-sample and two-tailed t-test, two-sided t-test. GeMSE also evaluates if the null hypothesis can be rejected accordint to a given $\alpha$ confidence, $p$-value, approximated degree of freedom, and homoscedasticity.

- Covariance and correlation: To spot correlation and dependencies, GeMSE allows performing covariance, Pearson product-moment correlation coefficient, and principle component analysis among genometric space row or columns.

GeMSE allows users to interactively choose a genometric space and an analysis to be performed, and to setup the required parameters; then, it visualizes data as single values (e.g., $p$-values) or plots, using scatter plots or heatmaps.

\section{Results}

We demonstrate the effective application and practical usefulness of GeMSE using 33 NGS Chromatin Immunoprecipitation sequencing (ChIP-seq) datasets from the Homo sapiens A549 immortalized cell line (an epithelial cell line derived from lung carcinoma tissue) [31], downloaded from ENCODE [3].

\section{A}

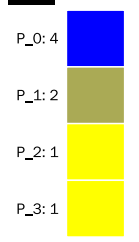

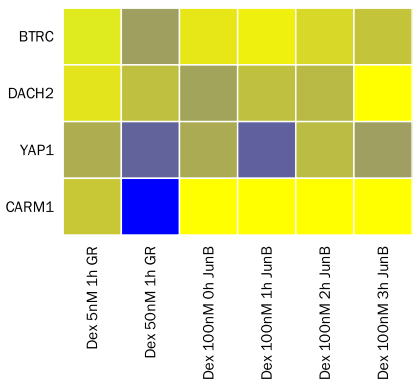

$\mathbf{B}$

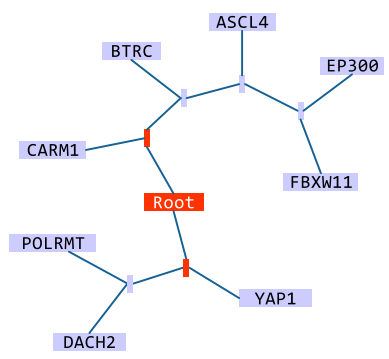

C

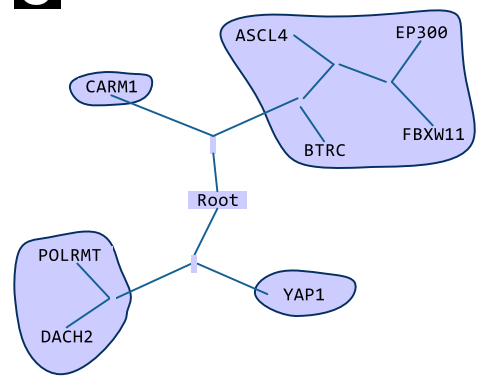

Fig. 5 Patterns exploration options: A heatmap, where each row represents a pattern and is labeled by the name of one of the elements of the pattern, and each column shows the counts of each of the patterns. B radial graph, where each node represents a vector (pattern analysis input), and edges are the relations between the nodes. Nodes colored red, are the nodes above the dendrogram cut, and nodes colored purple are below dendrogram cut; hence all the nodes colored purple after a red node, belong to the same pattern. C Force-directed graph, where nodes belonging to the same pattern are aggregated 


\section{Datasets}

The datasets used are summarized on Table 1; they cover various types of experiments, spanning different treatments and targeting various DNA-binding proteins.

- Some datasets belong to studies assessing the effect of treatments with Dexamethasone (Dex) on the DNA-binding enrichment profile of different proteins, including the treatments (a) with various doses of Dex (500 pM, 5 nM, and $50 \mathrm{nM}$ ) on NR3C1,

Table 1 Datasets of human A549 immortalized cell line used for GeMSE demonstration

\begin{tabular}{|c|c|c|c|c|c|}
\hline \# & Treatment & Dose & Duration & $\begin{array}{l}\text { Antibody } \\
\text { target }\end{array}$ & Replicates \\
\hline 1 & Dexamethasone & 500 pM & $1 \mathrm{~h}$ & NR3C 1 & $\bullet \bullet$ \\
\hline 2 & Dexamethasone & $5 \mathrm{nM}$ & $1 \mathrm{~h}$ & $\mathrm{NR3C1}$ & $\bullet \bullet$ \\
\hline 3 & Dexamethasone & $50 \mathrm{nM}$ & $1 \mathrm{~h}$ & NR3C1 & $\bullet \bullet$ \\
\hline 4 & Dexamethasone & $100 \mathrm{nM}$ & $30 \mathrm{~m}$ & JUNB & $\bullet \bullet$ \\
\hline 5 & Dexamethasone & $100 \mathrm{nM}$ & $\mathrm{Oh}$ & JUNB & $\bullet \bullet$ \\
\hline 6 & Dexamethasone & $100 \mathrm{nM}$ & $1 \mathrm{~h}$ & JUNB & $\bullet \bullet$ \\
\hline 7 & Dexamethasone & $100 \mathrm{nM}$ & $2 \mathrm{~h}$ & JUNB & $\bullet \bullet$ \\
\hline 8 & Dexamethasone & $100 \mathrm{nM}$ & $3 \mathrm{~h}$ & JUNB & $\bullet \bullet \bullet$ \\
\hline 9 & Dexamethasone & $100 \mathrm{nM}$ & $4 \mathrm{~h}$ & JUNB & $\bullet \bullet \bullet$ \\
\hline 10 & Dexamethasone & $100 \mathrm{nM}$ & $5 \mathrm{~h}$ & JUNB & $\bullet \bullet \bullet$ \\
\hline 11 & Dexamethasone & $100 \mathrm{nM}$ & $7 \mathrm{~h}$ & JUNB & $\bullet \bullet \bullet$ \\
\hline 12 & Dexamethasone & $100 \mathrm{nM}$ & $8 \mathrm{~h}$ & JUNB & $\bullet \bullet \bullet$ \\
\hline 13 & Dexamethasone & $100 \mathrm{nM}$ & $10 \mathrm{~h}$ & JUNB & $\bullet \bullet$ \\
\hline 14 & Dexamethasone & $100 \mathrm{nM}$ & $1 \mathrm{~h}$ & FOXA1 & $\bullet \bullet$ \\
\hline 15 & Dexamethasone & $100 \mathrm{nM}$ & $1 \mathrm{~h}$ & POLR2A & $\bullet \bullet$ \\
\hline 16 & Dexamethasone & $100 \mathrm{nM}$ & $1 \mathrm{~h}$ & USF1 & $\bullet \bullet$ \\
\hline 17 & Ethanol & $0.02 \%$ & $1 \mathrm{~h}$ & ATF3 & $\bullet \bullet \bullet$ \\
\hline 18 & Ethanol & $0.02 \%$ & $1 \mathrm{~h}$ & $\mathrm{BCL} 3$ & $\bullet \bullet$ \\
\hline 19 & Ethanol & $0.02 \%$ & $1 \mathrm{~h}$ & CTCF & $\bullet \bullet$ \\
\hline 20 & Ethanol & $0.02 \%$ & $1 \mathrm{~h}$ & EP300 & $\bullet \bullet$ \\
\hline 21 & Ethanol & $0.02 \%$ & $1 \mathrm{~h}$ & GABPA & $\bullet \bullet$ \\
\hline 22 & Ethanol & $0.02 \%$ & $1 \mathrm{~h}$ & JUND & $\bullet \bullet$ \\
\hline 23 & Ethanol & $0.02 \%$ & $1 \mathrm{~h}$ & POLR2A & $\bullet \bullet$ \\
\hline 24 & Ethanol & $0.02 \%$ & $1 \mathrm{~h}$ & REST & $\bullet \bullet$ \\
\hline 25 & Ethanol & $0.02 \%$ & $1 \mathrm{~h}$ & SIN3A & $\bullet \bullet$ \\
\hline 26 & Ethanol & $0.02 \%$ & $1 \mathrm{~h}$ & SIX5 & $\bullet \bullet$ \\
\hline 27 & Ethanol & $0.02 \%$ & $1 \mathrm{~h}$ & TAF1 & $\bullet \bullet$ \\
\hline 28 & Ethanol & $0.02 \%$ & $1 \mathrm{~h}$ & TCF12 & $\bullet \bullet$ \\
\hline 29 & Ethanol & $0.02 \%$ & $1 \mathrm{~h}$ & USF1 & $\bullet \bullet$ \\
\hline 30 & None & None & None & CTCF & $\bullet \bullet$ \\
\hline 31 & None & None & None & PBX3 & $\bullet \bullet$ \\
\hline 32 & None & None & None & RAD21 & $\bullet \bullet$ \\
\hline 33 & None & None & None & TEAD4 & $\bullet \bullet$ \\
\hline
\end{tabular}

a glucocorticoid receptor protein (see rows 1-3 on Tables 1 and 2), or (b) with $100 \mathrm{nM}$ of Dex on transcription factor jun-B for multiple durations ( $30 \mathrm{~m}, 0 \mathrm{~h}, 1 \mathrm{~h}, 2 \mathrm{~h}, 3 \mathrm{~h}, 4 \mathrm{~h}, 5 \mathrm{~h}, 7 \mathrm{~h}, 8 \mathrm{~h}$, and $10 \mathrm{~h}$; see rows 4-13 on Tables1 and 2), or (c) with $100 \mathrm{nM}$ of Dex for $1 \mathrm{~h}$ on different transcription factors (FOXA1, POLR2A, USF1; see rows 14-16 on Tables 1 and 2).

- Some other datasets belong to studies assessing the effect of $1 \mathrm{~h}$ treatment with $0.02 \%$ of Ethanol (EtOH) on different DNA-binding proteins (e.g., ATF-3, CTCF, jun-D; see rows 17-29 on Tables 1 and 2), or to studies assessing the activity of DNA-binding proteins under no treatment (see rows 30-33 on Tables 1 and 2).

\section{Data preparation}

Each dataset consists of 2-3 (isogenous) replicates. The replicates were comparatively evaluated using the Multiple Sample Peak Calling (MSPC) method [32], which locally lowers the minimum significance required to accept repeated evidences across replicates. We used MUSERA [33], a graphical implementation of the MSPC method, to combine multiple replicates of DNA-binding enriched region (i.e., called peak) samples of a dataset into a single sample without loosing or overestimating the significance of the called peak regions.

Each of the considered datasets has a target protein (summarized on Table 2). As the function of proteins tends to be regulated by other proteins (cf. interactomics), we used STRING [34] to search for proteinprotein interactions for each of the dataset target proteins. We found 163 proteins that interact with at least one of the dataset target proteins (see Fig. 6). We focused on these 182 proteins (i.e., 19 target proteins, and 163 proteins with which the target proteins interact).

As reference genomic regions, we used RefSeq [35] human gene annotations downloaded from Ensembl [36], focusing on those genes regarding the selected proteins based on gene name; we found 171 of them.

In GeMSE we loaded a reference sample with the considered genes, and the 33 replicate-combined ChIP-seq experiment samples obtained; thus, we mapped every DNA-protein binding enriched region in each of the latter samples on the considered genes (see flowchart and panel b on Fig. 1), and computed aggregate values of the attributes associated with the regions in each ChIP-seq sample that overlap each gene (i.e., region counts, averages of region $p$-values). In so doing, we built a genometric space $\mathrm{R}$ with 171 rows (genes) and 33 columns (samples/conditions) (see panel $\mathrm{R}$ on Fig. 7), which we fully explored and interactively analyzed by taking advantage of GeMSE. 
Table 2 Target proteins of the used datasets regarding treatments with Dexamethasone (Dex), or Ethanol (EtOH), or with no treatment (None)

\begin{tabular}{|c|c|c|c|c|c|c|}
\hline \# & Target protein & Antibody target & Associated disease (main) & Dex & $\mathrm{EtOH}$ & None \\
\hline 1 & Activating Transcription Factor 3 & ATF3 & Hodgkin's lymphoma [38] & & $\checkmark$ & \\
\hline 2 & B-cell lymphoma 3 & $\mathrm{BCL3}$ & Lymphoma and chronic lymphocytic leukemia [39] & & $\checkmark$ & \\
\hline 3 & Transcriptional repressor CTCF & CTCF & Regulation of chromatin architecture [40] & & $\checkmark$ & $\checkmark$ \\
\hline 4 & E1A binding protein $\mathrm{p} 300$ & EP300 & Rubinstein-Taybi syndrome [41] & & $\checkmark$ & \\
\hline 5 & Forkhead box protein A1 & FOXA1 & Estrogen receptor $\alpha(E R \alpha)$ breast cancer [42] & $\checkmark$ & & \\
\hline 6 & GA-binding protein alpha chain & GABPA & Down syndrome [43] & & $\checkmark$ & \\
\hline 7 & Transcription factor jun-B & JUNB & Myeloproliferative disorder [44] & $\checkmark$ & & \\
\hline 8 & Transcription factor jun-D & JUND & Adult-T cell leukaemia [45] & & $\checkmark$ & \\
\hline 9 & Glucocorticoid receptor & $\mathrm{NR3C} 1$ & Glucocorticoid resistance syndrome [46] & $\checkmark$ & & \\
\hline 10 & Pre-B-cell leukemia transcription factor 3 & PBX3 & Pilocytic astrocytoma [47] & & & $\checkmark$ \\
\hline 11 & DNA-directed RNA polymerase II subunit RPB1 & POLR2A & UV-sensitive syndrome [48] & $\checkmark$ & $\checkmark$ & \\
\hline 12 & Double-strand-break repair protein rad21 homolog & RAD21 & Cornelia de Lange syndrome [49] & & & $\checkmark$ \\
\hline 13 & RE1-silencing transcription factor & REST & Wilms tumor [50] & & $\checkmark$ & \\
\hline 14 & Paired amphipathic helix protein $\operatorname{Sin} 3 a$ & SIN3A & Chromosome 15q24 microdeletion syndrome [51] & & $\checkmark$ & \\
\hline 15 & Homeobox protein SIX5 & SIX5 & Branchio-oto-renal syndrome [52] & & $\checkmark$ & \\
\hline 16 & Transcription initiation factor TFIID subunit 1 & TAF1 & X-linked dystonia-parkinsonism [53] & & $\checkmark$ & \\
\hline 17 & Transcription factor 12 & TCF12 & Extraskeletal myxoid chondrosarcoma [54] & & $\checkmark$ & \\
\hline 18 & Transcriptional enhancer factor TEF-3 & TEAD4 & Narcolepsy [55] & & & $\checkmark$ \\
\hline 19 & Upstream stimulatory factor 1 & USF1 & Hyperlipidemia [56] & $\checkmark$ & $\checkmark$ & \\
\hline
\end{tabular}

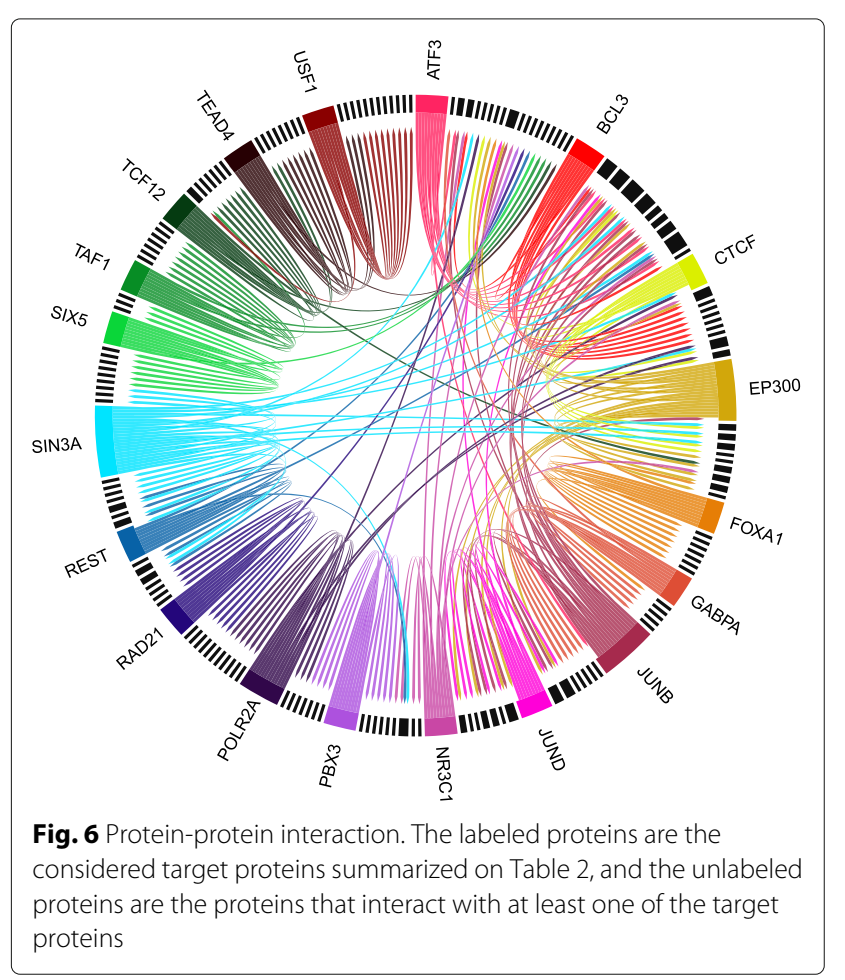

\section{Data exploration}

As an example, in our scenario GeMSE can be used to search for experiment samples with similar profiles of gene-protein binding enrichment significance. GeMSE can extract patterns of such profiles in the considered genometric space, leveraging on the following data transformation:

$$
R^{\prime}=\text { Cluster (C, AVERAGE, EU) } R
$$

In our case, GeMSE suggests the existence of 5 of such patterns (see panel Rpc on Fig. 7), and supports their explanation based on the metadata of samples sharing the same pattern (see Table 3). Referring to Table 3, all 10 jun-B samples with Dex $100 \mathrm{nM}$ treatment for various durations are grouped together in pattern $\mathrm{P}-1$, as well as both samples targeting POLR2A are in pattern P-2. These are interesting, yet expected, results that GeMSE highlights; answers to several other questions can be discovered through GeMSE. In the following subsections, we show how to discover more interesting aspects of the data by interactively exploring them taking advantage of the easy-to-use graphical interface for interactive analytics of GeMSE. 


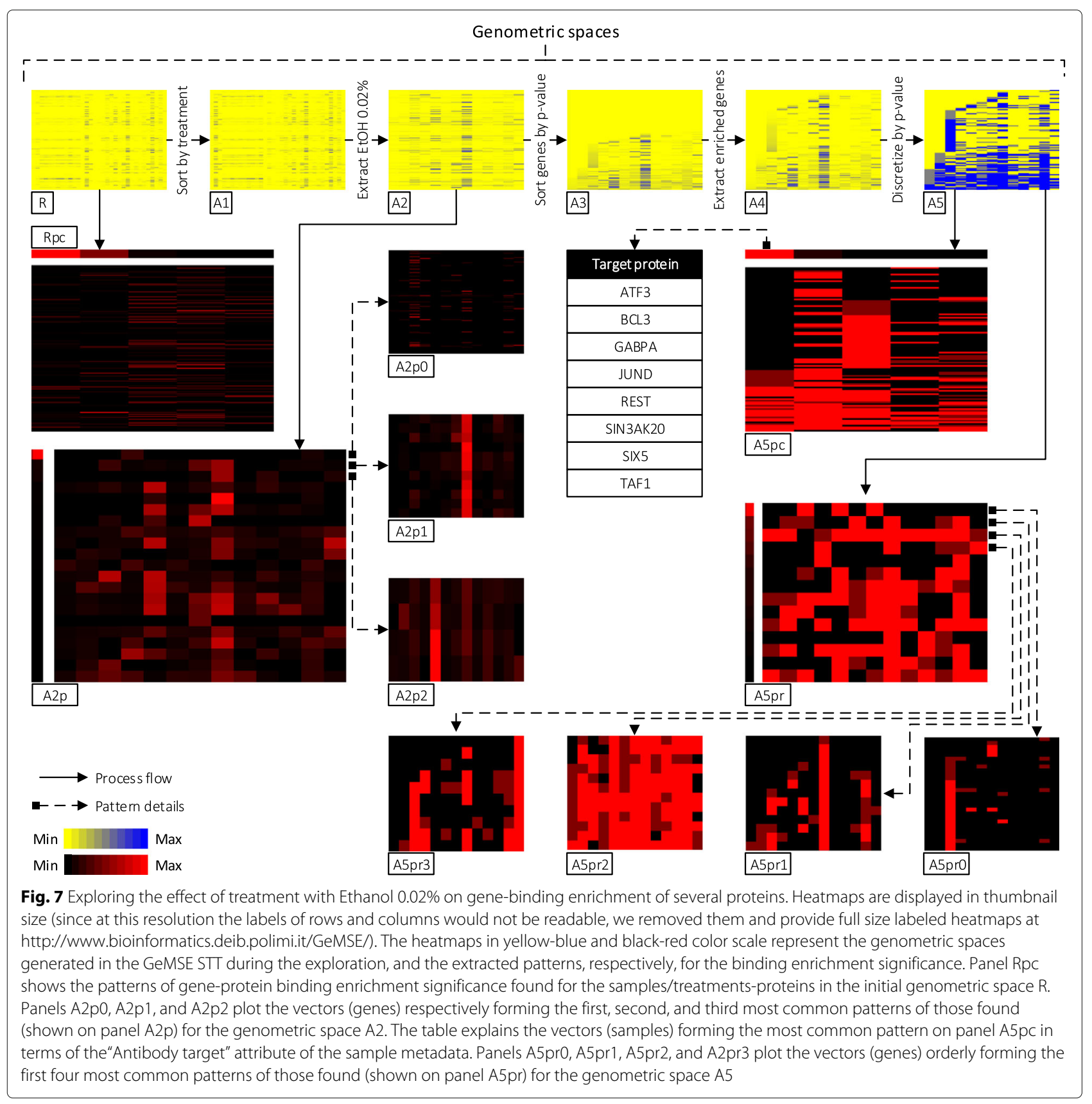

\section{Effects of Ethanol treatment}

In this subsection, we show how GeMSE can help in determining the effects of ethanol treatment on gene-protein binding enrichment profiles.

A number of considered input samples regard studying the effect of the treatment with Ethanol $0.02 \%$ on the DNA-binding enrichment profile of various proteins. To focus on these samples, in GeMSE we first sort data as follows (see panel A1 on Fig. 7):

$$
A 1=\text { Sort (C, ASCENDING, Treatment) } R
$$

By setting column labels of the heatmap to "Treatment", and looking at the heatmap or at the grid view (a tabular representation of a genometric space in GeMSE) of the result, we see that obtained columns 16-28 represent samples with EtOH $0.02 \%$ treatment; we extract these columns as follows (see panel A2 on Fig. 7):

$$
A 2=\operatorname{Extract}([16,29),[0,171)) A 1
$$

Then, we search for patterns of gene-protein binding enrichment significance only on the extracted samples 
Table 3 Excerpt of metadata aggregation for the five patterns of gene-protein binding enrichment significance that correspond to the root of the STT of the performed data exploration described in Fig. 7 (see panel Rpc on Fig. 7)

\begin{tabular}{lllllll}
\hline Attribute & Value & P-0 & P-1 & P-2 & P-3 & P-4 \\
\hline Antibody target & POLR2A & 0 & 0 & 2 & 0 & 0 \\
Treatment & Dex 100 nM 30 m & 0 & 1 & 0 & 0 & 0 \\
Treatment & Dex 100 nM 0 h & 0 & 1 & 0 & 0 & 0 \\
Treatment & Dex 100 nM 1 h & 0 & 1 & 0 & 0 & 0 \\
Treatment & Dex 100 nM 2h & 0 & 1 & 0 & 0 & 0 \\
Treatment & Dex 100 nM 3h & 0 & 1 & 0 & 0 & 0 \\
Treatment & Dex 100 nM 4h & 0 & 1 & 0 & 0 & 0 \\
Treatment & Dex 100 nM 5h & 0 & 1 & 0 & 0 & 0 \\
Treatment & Dex 100 nM 7h & 0 & 1 & 0 & 0 & 0 \\
Treatment & Dex 100 nM 8h & 0 & 1 & 0 & 0 & 0 \\
Treatment & Dex 100 nM 10 h & 0 & 1 & 0 & 0 & 0 \\
\hline
\end{tabular}

with $\mathrm{EtOH} 0.02 \%$ treatment; this can be done by clustering the obtained genometric space A2 by rows/genes (instead of by columns/samples, as in the initial example). Leveraging on the following data transformation:

$$
A 2^{\prime}=\text { Cluster (R, AVERAGE, EU) } A 2
$$

GeMSE suggests 21 patterns (see Fig. 8 for Elbow method data), each representing a group of genes with similar profiles of gene-protein binding enrichment significance for the extracted $\mathrm{EtOH} 0.02 \%$ treatment samples (see panel A2p on Fig. 7).

GeMSE allows further exploration of each of the extracted patterns, by expanding a pattern to the individual elements it groups (in this case, genes) and visualizing on a heatmap the values of the element associated attribute considered (in this case, binding enrichment significance $p$-value for each of the grouped genes and each evaluated sample). For instance, the three upper most

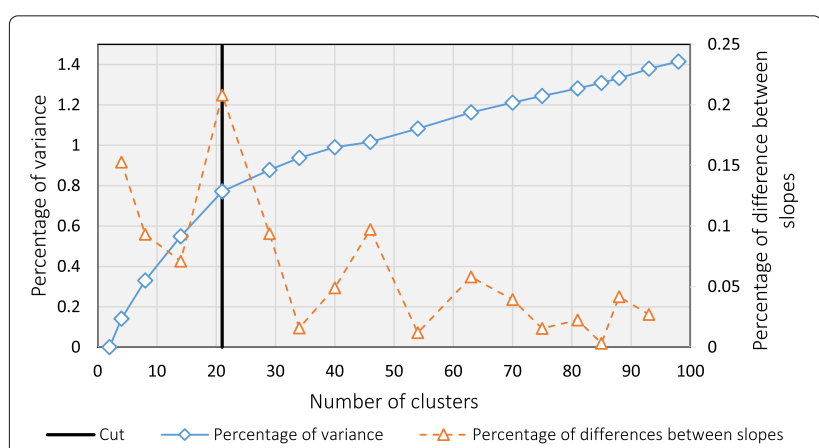

Fig. 8 Application of the Elbow method for finding the optimal number of clusters on A2 genometric space of Fig. 7. Based on this method, the optimal number of clusters is 21 patterns in panel A2p on Fig. 7 are expanded to the contributing genes and plotted on panels A2p0, A2p1, and A2p 2 on Fig. 7. A pattern can also be described by using the metadata of the elements it groups; e.g., the left most pattern in panel A5pc on Fig. 7 is described in the table on Fig. 7 using sample metadata. Additionally, GeMSE allows using any of the numerical attributes associated with the pattern elements (e.g., in our case, $p$-value, q-value, region count) for visualization of the individual components of a pattern. This allows assessing patterns based on various quantifying attributes.

Popularity of a pattern within a dataset (i.e., number of dataset elements sharing the pattern) can also be easily observed. Combining such observation with the intensity of the attribute values associated with the pattern elements can provide useful support for further evaluations. For example, the upper most pattern in panel A2p on Fig. 7 is very common (117 out of 171 genes share it; see panel $\mathrm{A} 2 \mathrm{p} 0$ on Fig. 7). Yet, it is formed by genes with no or weak protein binding enrichment across all samples; thus, we may not be interested in such pattern. We may also exclude the genes with low or no protein binding enrichment on all samples; such gene filtering can be done as follows.

We can first sort the rows/genes of the genometric space in ascending order (instead of sorting by columns/samples, as previously done) as follows (see panel A3 on Fig. 7):

$$
A 3=\text { Sort (R, ASCENDING, } p \text {-value) } A 2
$$

Then, using a grid view we look at the sorted genometric space A3 and identify the row $r$ (with $r=72$, in our case) as the first row/gene with protein binding enrichment in at least one of the samples (i.e., all the genes at rows before $r$ have protein binding enrichment in neither of the samples, and all the genes on and after $r$ have protein binding enrichment at least in one of the samples). Then, we extract rows from $r$ to the last row of the ordered genometric space A3 as follows (see panel A4 on Fig. 7):

$$
A 4=\operatorname{Extract}([0,13),[72,171)) A 3
$$

Even after removing genes with no protein binding enrichment, some of the remaining genes may have a low significant protein binding enrichment, while some others may be highly significantly enriched. It may then be useful to discretize gene-protein binding enrichment significance as weak, middle, or strong. Given the nature of significance $p$-values, it may be worth grouping together data with $p$-values $1 \cdot \mathrm{e}^{-12}$ (significant) and $1 \cdot \mathrm{e}^{-200}$ (very significant), rather than grouping data with $p$-values $1 \cdot \mathrm{e}^{-12}$ (significant) and $1 \cdot \mathrm{e}^{-4}$ (low significant), as it would probably occur by $p$-value clustering. This is obtained by the Discretize transformation, applied on A4 as it follows, where the $p$-values 40 and 80 are in $-10 \cdot \log _{10}$ format 
as in the A4 data (see panel A5 on Fig. 7):

$A 5=$ Discretize $([0,13),[0,99),[40,0,1],[80,1,2]) A 4$

On the discretized genometric space A5, we can search for genes with similar pattern of protein binding enrichment significance, and find the patterns in panel A5pr on Fig. 7. We note that:

1. The most common pattern found (see panel A5pr0 on Fig. 7) includes genes with significant protein binding enrichment in samples targeting CTCF or REST proteins (row number 19 and 23 on Table 1).

2. The second most common pattern (see panel A5pr1 on Fig. 7) includes genes that have mostly significant enrichment of POLR2A binding (sample in row number 24 on Table 1 ).

3. The third most common pattern (see panel A5pr2 on Fig. 7) includes genes with significant protein binding enrichment in most of the samples.

4. The forth most common pattern (see panel A5pr3 on Fig. 7) includes the set of genes with mostly significant enrichment of USF1 binding (sample in row 29 on Table 1 ).

Then, on the discretized genometric space A5, we can also search for samples with similar pattern of protein binding enrichment significance. Based on the GeMSE suggested number of clusters, we find 5 patterns (see panel A5pc on Fig. 7), with one of them in common among 8 out of 13 samples with EtOH $0.02 \%$ treatment. We use GeMSE to explore this pattern, and choose to see the values of the "Antibody target" metadata attribute of the samples with this pattern; this operation lists all the target proteins of such samples (see the table on Fig. 7), which include proteins SIN3A and REST. This finding might have several interpretations; for instance, an explanation could be that the REST transcription factor is known to repress transcription by recruiting the corepressor SIN3A [37].

\section{Effects of Dexamethasone}

Here, we show how various doses of Dexamethasone treatment affect gene-binding enrichment of the NR3C1 protein, by using GeMSE on the considered data. Solving this problem requires a data exploration procedure different from the previously performed one, starting from the genometric space A1 obtained after the sort by treatment operation at the beginning of the previously described data exploration, and ready available in the GeMSE STT (see Fig. 3). As our considered data include three samples targeting the $\mathrm{NR} 3 \mathrm{C} 1$ protein and regarding Dexamethasone treatment with $500 \mathrm{pM}, 5 \mathrm{nM}$ and $50 \mathrm{nM}$ dose, respectively, first we want to extract their corresponding columns from A1. After looking at the A1 heatmap and identifying the required columns as columns $13-15$ in A1, we extract them as follows:

$$
B 1=\operatorname{Extract}([13,16),[0,171)) A 1
$$

Then, we remove genes without protein binding enrichment as done in previous exploration, i.e., through gene sorting by enrichment $p$-value, visual inspection of the heatmap of the sorted genometric space B2 obtained, identification of the row $r$ in B2 corresponding to the first gene with NR3C1 binding enrichment in at least one of the samples (i.e., $r=130$, in our case), and extraction of the rows from $r$ to the last row of the ordered genometric space B2 (see panel B3 on Fig. 9).

Then, we search for patterns of gene-binding enrichment significance across the three samples. GeMSE suggests 2 patterns of enrichment significance for the gene-binding of the $\mathrm{NR} 3 \mathrm{C} 1$ protein (see panel $\mathrm{B} 3 \mathrm{pc}$ on Fig. 9), where the binding enrichment significance for the treatment with $50 \mathrm{nM}$ of Dex are in a separate group from the significance regarding the treatments with lower doses of Dex, i.e., $5 \mathrm{nM}$ and $500 \mathrm{pM}$. This can be seen also from the metadata aggregation table that GeMSE provides (see an excerpt of it in the table on Fig. 9).

\section{Conclusions}

The availability of huge, well-curated and open repositories of processed genomic datasets motivates our efforts
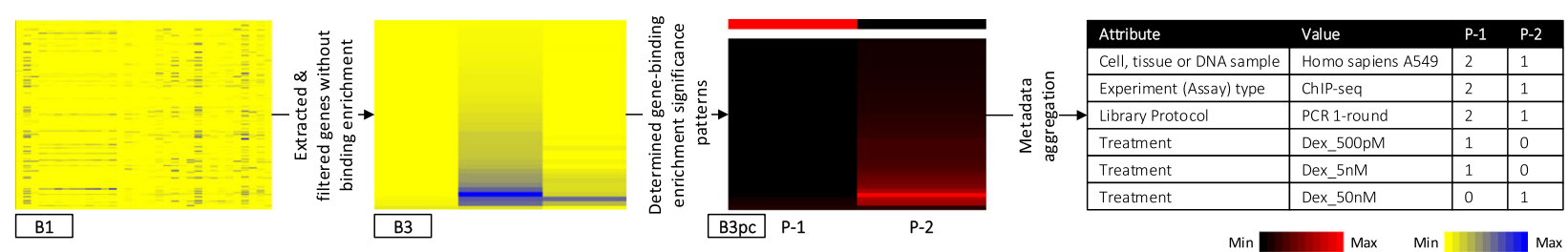

Fig. 9 Exploring the effect of treatment with various doses of Dexamethasone on the enrichment of the gene-binding of the NR3C1 protein. From the initial panel B1, three columns corresponding to samples regarding treatments with different Dexamethasone doses (500 pM, $5 \mathrm{nM}$ and $50 \mathrm{nM})$ are extracted, and from them genes without binding enrichment are removed (panel B3); finally, patterns of gene-binding enrichment significance are extracted in panel B3pc. Metadata aggregations of the two identified patterns are shown in the table. Heatmaps at full size and with their row/column labels are available at http://www.bioinformatics.deib.polimi.it/GeMSE/ 
in designing and implementing new data exploration abstractions, so as to facilitate effective biological knowledge discovery through interactive analytics.

Thanks to the notion of genometric space, our GeMSE tool at the same time rises the expressive power of userdata interaction and lowers the complexity of data exploration, making it available to nonprogrammers. The tool supports a trial-and-error approach that can be very useful for both defining the appropriate knowledge extraction pipelines and exploring alternative hypotheses, making GeMSE a relevant interactive analytics application.

GeMSE effectively provides the tracing of data exploration steps through a state-transition diagram, whose states, which represent exploration step results, are all accessible at any time; this is obtained thanks to an efficient algorithm for state-transition caching and reconstruction implemented in the tool. Evolution of the data exploration occurs by means of state transitions which embody genometric space transformations.

GeMSE effective application and practical usefulness is demonstrated through significant use cases of biological interest.

\section{Availability and requirements \\ Project name: GeMSE \\ Project homepage: http://www.bioinformatics.deib.} polimi.it/GeMSE/

Project source code and discussions and issues page: https://github.com/Genometric/GeMSE

License: GPL v3.0

Operating system(s): platform independent (tested on Microsoft Windows 10, macOS Sierra, and Ubuntu 16).

Programming language: Java

Other requirements: if chosen to connect with $R$, GeMSE requires $R$ installation with gplots, ape, and hclust packages installed.

Tutorial and example data: available at project homepage.

\section{Abbreviations}

BED: Browser extensible data; CDS: Coding sequence; CSV: Comma-separated value; Dex: Dexamethasone; DNA: Deoxyribo nucleic acid; ENCODE: Encyclopedia of DNA elements; EtOH: Ethanol; GeMSE: GenoMetric space explorer; GMQL: GenoMetric query language; GTF: General transfer format; IA: Interactive analytics; MSPC: Multiple sample peak calling; MuSERA: Multiple sample enrichment region assessment; NGS: Next generation sequencing; STRING: Search tool for the retrieval of interacting genes; STT: State-transition tree; TCGA: The cancer genome atlas; UCSC: University of california santa cruz; UTR: UnTranslated region

\section{Acknowledgements}

We thank Fernando Palluzzi, Mohammad Heydarian, Shamilene Sivagnanam, and Luke Sargent for their comments on the manuscript, implemented tool, and use-cases.

\section{Funding}

This work was supported by the Advanced ERC Grant "Data-Driven Genomic Computing (GeCo)" project (2016-2021), funded by the European Research Council, which covered also the publication costs.

\section{Authors' contributions}

VJ: Designed, developed and tested the GeMSE tool, designed and performed use-cases, and wrote this manuscript. MMat: Contributed to design the implemented tool and reviewed this manuscript. MMas: Contributed to design the implemented tool and reviewed this manuscript. SC: Supervised the project and initiated the idea of the tool and conceived the project, contributed to design the implemented tool and use-cases, and revised the manuscript critically. All authors read and approved the final manuscript.

\section{Ethics approval and consent to participate}

Not applicable.

\section{Consent for publication}

Not applicable.

\section{Competing interests}

The authors declare that they have no competing interests.

\section{Publisher's Note}

Springer Nature remains neutral with regard to jurisdictional claims in published maps and institutional affiliations.

Received: 18 June 2017 Accepted: 19 November 2017

Published online: 04 December 2017

\section{References}

1. Luu PL, Gerovska D, Arrospide-Elgarresta M, Retegi-Carrión S, Schöler HR, Araúzo-Bravo MJ. P3BSseq: parallel processing pipeline software for automatic analysis of bisulfite sequencing data. Bioinformatics. 2017;33(3):428-31.

2. Wolfien $M$, Rimmbach $C$, Schmitz $U$, Jung JJ, Krebs S, Steinhoff $G$, David R, Wolkenhauer O. TRAPLINE: a standardized and automated pipeline for RNA sequencing data analysis, evaluation and annotation. BMC Bioinformatics. 2016;17:21

3. ENCODE Project Consortium. An integrated encyclopedia of DNA elements in the human genome. Nature. 2012;489(7414):57-74

4. Romanoski CE, Glass CK, Stunnenberg HG, Wilson L, Almouzni G. Epigenomics: Roadmap for regulation. Nature. 2015;518(7539):314-6.

5. Cancer Genome Atlas Research Network, Weinstein JN, Collisson EA, Mills GB, Shaw KRM, Ozenberger BA, Ellrott K, Shmulevich I, Sander C, Stuart JM. The Cancer Genome Atlas Pan-Cancer analysis project. Nat Genet. 2013;45(10):1113-20.

6. 1000 Genomes Project Consortium, Abecasis GR, Altshuler D, Auton A, Brooks LD, Durbin RM, Gibbs RA, Hurles ME, McVean GA. A map of human genome variation from population-scale sequencing. Nature. 2010;467(7319):1061-73.

7. Masseroli M, Pinoli P, Venco F, Kaitoua A, Jalili V, Palluzzi F, Muller H, Ceri S. GenoMetric Query Language: a novel approach to large-scale genomic data management. Bioinformatics. 2015;31(12):1881-8.

8. Rutherford K, Parkhill J, Crook J, Horsnell T, Rice P, Rajandream MA, Barrell B. Artemis: sequence visualization and annotation. Bioinformatics. 2000;16(10):944-5.

9. Kent WJ, Sugnet CW, Furey TS, Roskin KM, Pringle TH, Zahler AM, Haussler D. The human genome browser at UCSC. Genome Res. 2002;12(6):996-1006.

10. Eisen MB, Spellman PT, Brown PO, Botstein D. Cluster analysis and display of genome-wide expression patterns. Proc Natl Acad Sci. 1998;95(25):14863-8.

11. Gould J.GENE-E. http://www.broadinstitute.org/cancer/software/GENE-E/. Accessed 7 June 2017.

12. Gentleman RC, Carey VJ, Bates DM, Bolstad B, Dettling M, Dudoit S, Ellis B, Gautier L, Ge Y, Gentry J, Hornik K, Hothorn T, Huber W, lacus S, Irizarry R, Leisch F, Li C, Maechler M, Rossini AJ, Sawitzki G, Smith C, Smyth G, Tierney L, Yang JY, Zhang J. Bioconductor: open software development for computational biology and bioinformatics. Genome Biol. 2004;5(10):1.

13. Ye T, Krebs AR, Choukrallah MA, Keime C, Plewniak F, Davidson I, Tora L. seqMINER: an integrated ChIP-seq data interpretation platform. Nucleic Acids Res. 2011;39(6):35-5.

14. Loh Y-HE, Shen L. Analysis and visualization of ChIP-seq and RNA-seq sequence alignments using ngs.plot. Methods Mol Biol. 2016;1415: 371-383. doi:10.1007/978-1-4939-3572-7_18. 
15. Khomtchouk BB, Hennessy JR, Wahlestedt C. Microscope: ChIP-seq and RNA-seq software analysis suite for gene expression heatmaps. BMC Bioinformatics. 2016;17(1):390.

16. Goecks J, Nekrutenko A, Taylor J, Team TG. Galaxy: a comprehensive approach for supporting accessible, reproducible, and transparent computational research in the life sciences. Genome Biol. 2010;11(8):86

17. Höllt T, Pezzotti N, van Unen V, Koning F, Eisemann E, Lelieveldt B, Vilanova A. Cytosplore: Interactive immune cell phenotyping for large single-cell datasets. In: Computer Graphics Forum. 2016. p. 171-80. doi:10.1111/cgf.12893.

18. Goecks J, Coraor N, Nekrutenko A, Taylor J, Team G. NGS analyses by visualization with Trackster. Nat Biotechnol. 2012;30(11):1036-9.

19. O'neil EJ, O'neil PE, Weikum G. The Iru-k page replacement algorithm for database disk buffering. ACM SIGMOD Record. 1993;22(2):297-306.

20. Hclust. https://www.rdocumentation.org/packages/fastcluster/versions/ 1.1.22/topics/hclust. Accessed 7 June 2017

21. Gplots. https://www.rdocumentation.org/packages/gplots/versions/3.0.1. Accessed 7 June 2017.

22. Wikipedia. Newick Format. https://en.wikipedia.org/wiki/Newick_format. Accessed 7 June 2017.

23. Heer J, Card SK, Landay JA. Prefuse: a toolkit for interactive information visualization. In: Proceedings of the SIGCHI Conference on Human Factors in Computing Systems. New York: ACM. 2005. p. 421-30. doi:10.1145/1054972.1055031.

24. Thorndike RL. Who belongs in the family? Psychometrika. 1953;18(4): 267-76.

25. Tibshirani R, Walther G, Hastie T. Estimating the number of clusters in a data set via the gap statistic. J Royal Stat Soc Ser B (Stat Method). 2001;63(2):411-23.

26. Milligan GW, Cooper MC. An examination of procedures for determining the number of clusters in a data set. Psychometrika. 1985;50(2):159-79.

27. Cios KJ, Pedrycz W, Swiniarski RW. Data mining and knowledge discovery. In: Data Mining Methods for Knowledge Discovery. New York: Springer. 1998. p. 1-26.

28. Karypis G, Han EH, Kumar V. Chameleon: Hierarchical clustering using dynamic modeling. Computer. 1999;32(8):68-75.

29. Akaike $\mathrm{H}$. Information theory and an extension of the maximum likelihood principle. In: Selected Papers of Hirotugu Akaike. New York: Springer. 1998. p. 199-213.

30. Schwarz G. Estimating the dimension of a model. Annals Stat. 1978;6(2): 461-4.

31. Lieber M, Smith B, Szakal A, Nelson-Rees W, Todaro G. A continuous tumor-cell line from a human lung carcinoma with properties of type II alveolar epithelial cells. Int J Cancer. 1976;17(1):62-70.

32. Jalili V, Matteucci M, Masseroli M, Morelli MJ. Using combined evidence from replicates to evaluate ChIP-seq peaks. Bioinformatics. 2015;31(17): 2761-9.

33. Jalili V, Matteucci M, Morelli MJ, Masseroli M. MuSERA: Multiple Sample Enriched Region Assessment. Brief Bioinform. 2017;18(3):367-81.

34. Szklarczyk D, Franceschini A, Wyder S, Forslund K, Heller D, Huerta-Cepas J, Simonovic M, Roth A, Santos A, Tsafou KP, Kuhn M, Bork $P$, Jensen L, von Mering C. STRING v10: protein-protein interaction networks, integrated over the tree of life. Nucleic Acids Res. 2015:43(Database issue):447-52.

35. Pruitt KD, Brown GR, Hiatt SM, Thibaud-Nissen F, Astashyn A, Ermolaeva O, Farrell CM, Hart J, Landrum MJ, McGarvey KM, Murphy MR, O'Leary NA, Pujar S, Rajput B, Rangwala SH, Riddick LD, Shkeda A, Sun H, Tamez P, Tully RE, Wallin C, Webb D, Weber J, Wu W, DiCuccio M, Kitts P, Maglott DR, Murphy TD, Ostell JM. RefSeq: an update on mammalian reference sequences. Nucleic Acids Res. 2014;42(Database issue):756-63.

36. Aken BL, Ayling S, Barrell D, Clarke L, Curwen V, Fairley S, Fernandez Banet J, Billis K, García Girón C, Hourlier T, Howe K, Kähäri A, Kokocinski F, Martin FJ, Murphy DN, Nag R, Ruffier M, Schuster M, Tang YA, Vogel JH, White S, Zadissa A, Flicek P, Searle SM. The Ensembl gene annotation system. Database. 2016;2016:093.

37. Huang Y, Myers SJ, Dingledine R. Transcriptional repression by REST: recruitment of $\operatorname{Sin} 3 \mathrm{~A}$ and histone deacetylase to neuronal genes. Nature Neurosci. 1999;2:867-72.

38. Janz M, Hummel M, Truss M, Wollert-Wulf B, Mathas S, Jöhrens K, Hagemeier C, Bommert K, Stein H, Dörken B, Bargou RC. Classical
Hodgkin lymphoma is characterized by high constitutive expression of activating transcription factor 3 (ATF3), which promotes viability of Hodgkin/Reed-Sternberg cells. Blood. 2006;107(6):2536-9.

39. Westerheide SD, Mayo MW, Anest V, Hanson JL, Baldwin AS. The putative oncoprotein $\mathrm{BCl}-3$ induces cyclin D1 to stimulate $\mathrm{G} 1$ transition. Mol Cell Biol. 2001;21(24):8428-36.

40. Phillips JE, Corces VG. CTCF: master weaver of the genome. Cell. 2009;137(7):1194-211.

41. Roelfsema JH, White SJ, Ariyürek Y, Bartholdi D, Niedrist D, Papadia F, Bacino CA, den Dunnen JT, van Ommen G-JB, Breuning MH, Hennekam RC, Peters DJ. Genetic heterogeneity in Rubinstein-Taybi syndrome: mutations in both the CBP and EP300 genes cause disease. Am J Human Genet. 2005;76(4):572-80.

42. Zhang MH, Man HT, Zhao XD, Dong N, Ma SL. Estrogen receptor-positive breast cancer molecular signatures and therapeutic potentials (Review). Biomed Reports. 2014;2(1):41-52.

43. Malinge S, Izraeli S, Crispino JD. Insights into the manifestations, outcomes, and mechanisms of leukemogenesis in Down syndrome. Blood. 2009;113(12):2619-28.

44. Passegué E, Wagner EF, Weissman IL. Junb deficiency leads to a myeloproliferative disorder arising from hematopoietic stem cells. Cell. 2004;119(3):431-43.

45. Kuhlmann AS, Villaudy J, Gazzolo L, Castellazzi M, Mesnard JM, Dodon MD HTLV-1 HBZ cooperates with JunD to enhance transcription of the human telomerase reverse transcriptase gene (hTERT). Retrovirology. 2007;4(1):92

46. Donner KM, Hiltunen TP, Jänne OA, Sane T, Kontula K. Generalized glucocorticoid resistance caused by a novel two-nucleotide deletion in the hormone-binding domain of the glucocorticoid receptor gene NR3C1. European J Endocrinol. 2013;168(1):9-18.

47. Ho CY, Bar E, Giannini C, Marchionni L, Karajannis MA, Zagzag D, Gutmann DH, Eberhart CG, Rodriguez FJ. MicroRNA profiling in pediatric pilocytic astrocytoma reveals biologically relevant targets, including PBX3, NFIB, and METAP2. Neuro Oncol. 2013;15(1):69-82.

48. POLR2A Gene. http://www.genecards.org/cgi-bin/carddisp.pl?gene= POLR2A. Accessed 7 June 2017.

49. Revenkova E, Focarelli ML, Susani L, Paulis M, Bassi MT, Mannini L, Frattini A, Delia D, KrantzI, Vezzoni P, Jessberger R, Musio A. Cornelia de Lange syndrome mutations in SMC1A or SMC3 affect binding to DNA. Hum Mol Genet. 2009;18(3):418-27.

50. Mahamdallie SS, Hanks S, Karlin KL, Zachariou A, Perdeaux ER, Ruark E, Shaw CA, Renwick A, Ramsay E, Yost S, Elliott A, Birch J, Capra M, Gray J, Hale J, Kingston J, Levitt G, McLean T, Sheridan E, Renwick A, Seal S, Stiller C, Sebire N, Westbrook TF, Rahman N. Mutations in the transcriptional repressor REST predispose to Wilms tumor. Nat Genet. 2015;47(12):1471-4.

51. Magoulas PL, El-Hattab AW. Chromosome 15q24 microdeletion syndrome. Orphanet J Rare Dis. 2012;7(1):2.

52. Hoskins BE, Cramer CH, Silvius D, Zou D, Raymond RM, Orten DJ, Kimberling WJ, Smith RJ, Weil D, Petit C, Otto EA, Xu PX, Hildebrandt F. Transcription factor SIX 5 is mutated in patients with branchio-oto-renal syndrome. Am J Human Genet. 2007;80(4):800-4.

53. Evidente VGH. X-linked Dystonia-Parkinsonism. Seattle: University of Washington; 2015.

54. Sjögren H, Wedell B, Kindblom JMM, Kindblom LG, Stenman G. Fusion of the $\mathrm{NH}$ 2-terminal domain of the basic helix-loop-helix protein TCF12 to TEC in extraskeletal myxoid chondrosarcoma with translocation $\mathrm{t}(9 ; 15)(\mathrm{q} 22 ; \mathrm{q} 21)$. Cancer Res. 2000;60(24):6832-5.

55. Luca G, Haba-Rubio J, Dauvilliers Y, Lammers GJ, Overeem S, Donjacour CE, Mayer G, Javidi S, Iranzo A, Santamaria J, Peraita-Adrados R, Hor H, Kutalik Z, Plazzi G, Poli F, Pizza F, Arnulf I, Lecendreux M, Bassetti C, Mathis J, Heinzer R, Jennum P, Knudsen S, Geisler P, Wierzbicka A, Feketeova E, Pfister C, Khatami R, Baumann C, Tafti M, European Narcolepsy Network. Clinical, polysomnographic and genome-wide association analyses of narcolepsy with cataplexy: a European Narcolepsy Network study. J Sleep Res. 2013;22(5):482-95.

56. Pajukanta P, Lilja HE, Sinsheimer JS, Cantor RM, Lusis AJ, Gentile M, Duan XJ, Soro-Paavonen A, Naukkarinen J, Saarela J, Laakso M, Ehnholm C, Taskinen MR, Peltonen L. Familial combined hyperlipidemia is associated with upstream transcription factor 1 (USF1). Nat Genet. 2004;36(4):371-6. 